rev Psi

Revista de Psicología (UNLP)

https://revistas.unlp.edu.ar/revpsi

\title{
Violencia e identidad de género: consideraciones sobre las niñas desde el campo de la salud
}

\author{
María Manuela Kreis ${ }^{1,2}$ \\ Agustina María Edna D’Agostino²
}

\section{Correspondencia}

manu.kreis@gmail.com

Filiaciones institucionales

${ }^{1}$ Consejo Nacional de Investigaciones Científicas $\quad{ }^{2}$ Laboratorio de Psicología Comunitaria y Políticas y Técnicas (CONICET, Argentina) Públicas, Facultad de Psicología, Universidad

Nacional de La Plata (UNLP, Argentina)

\section{Resumen}

En el presente trabajo nos proponemos introducir algunas relaciones entre la dimensión histórico-social, las problemáticas actuales vinculadas a la violencia e identidad de género en niñas y el proceso de salud-enfermedad-atención para las feminidades. A partir de aquí, señalamos que la particularización de las niñas se vinculó históricamente a estrategias de saber-poder y a universos de significaciones que naturalizaron las desigualaciones de género desde la temprana edad. Asimismo, observamos que la violencia de género constituye una problemática actual que puede tener un impacto en la salud de las niñas. Por último, advertimos que junto a la emergencia de identidades de género en la niñez, como las niñas trans, coexisten prácticas cisexistas en los dispositivos de salud que es necesario interrogar.

\section{Palabras clave}

niñas | género | violencias | salud mental

\section{Cómo citar}

Kreis, M. M. y D’Agostino, A. M. E. (2021).

Violencia e identidad de género: consideraciones sobre las niñas desde el campo de la salud. Revista de Psicología, 20(2), 89-105. HTTPS://DX.DOI.ORG/ $10.24215 / 2422572$ XE098

\begin{tabular}{lll}
\multicolumn{2}{l}{$\begin{array}{l}\text { Proceso editorial } \\
\text { Recibido }\end{array}$} & ISSN \\
11 nov. 2020 & 23 mar. 2021 & $2422-572 \mathrm{X}$ \\
Aceptado & Publicado & Licencia \\
6 jun. 2021 & 9 jun. 2021 & $\begin{array}{l}\text { Licencia de Cultura Libre CC-BY 4.0 } \\
\text { (Compartir - Adaptar - Atribuir) }\end{array}$ \\
Editor & Entidad editora \\
Nicolás Alessandroni (Facultad de Psicología & RevPsi es una publicación de la \\
UAM, España / Facultad de Psicología UNLP, & Facultad de Psicología (Universidad \\
Argentina). & Nacional de La Plata, Argentina)
\end{tabular}




\section{Violência e identidade de gênero: considerações sobre meninas na área da saúde}

\section{Resumo}

O presente trabalho tem por objetivo apresentar algumas relações entre a dimensão histórico-social, os problemas atuais relacionados à violência e identidade de gênero em meninas e o processo saúde-doença-cuidado para a feminilidade. A partir daqui, apontamos que a particularização das meninas esteve historicamente ligada a estratégias de saber-poder e a universos de significados que naturalizaram as desigualdades de gênero desde a infância. Da mesma forma, observamos que a violência de gênero constitui um problema atual que pode impactar a saúde das meninas. Por fim, constatamos que junto com o surgimento das identidades de gênero na infância, como as meninas trans, coexistem práticas cisexistas nas unidades de saúde que precisam ser questionadas.

\section{Palavras-chave}

meninas | gênero | violência | saúde mental

\section{Violence and gender identity: considerations about girls in the area of health}

\section{Abstract}

The aim of this article is to introduce some relationships between the historical-social dimension, the current problems related to violence and gender identity in girls, and the health-disease-care process for feminities. From here, we point out that the particularization of girls was historically linked to knowledge-power strategies and to universes of meanings that naturalized gender inequalities from an early age. Likewise, we observe that gender-based violence constitutes a current problem that can have an impact on the health of girls. Finally, we note that along with the emergence of gender identities in childhood, such as trans girls, cissexist practices coexist in health care facilities that need to be questioned.

\section{Keywords}

girls | gender | violence | mental health 


\section{Aspectos destacados del trabajo}

- La particularización de las niñas respondió a estrategias de saber-poder que naturalizaron desigualaciones de género.

- Los criterios de salud mental para las mujeres partieron del modo de subjetivación femenino tradicional.

- La violencia de género constituye una problemática presente en el proceso de salud-enfermedad-cuidado de las mujeres.

- La emergencia de producciones subjetivas en niñas requiere decisexualizar el abordaje de la salud mental.

Las problemáticas del campo de la salud mental requieren tener en cuenta el contexto social, económico, cultural y las particularidades de los atravesamientos poblacionales. Respecto a la salud mental en la niñez, distintas investigaciones señalan que las demandas que se presentan en los servicios, constituyen problemáticas complejas en las que los cuadros psicopatológicos están imbricados con condiciones de vulneración social y económica (Stolkiner, 2019; Barcala, 2019). Asimismo, se identifica la emergencia de nuevas demandas en niñez, como las de acompañamiento de cambio de identidad de género (Tajer, 2017).

Ante esto, se observa que, en algunas oportunidades, los/as profesionales interpretan en términos psicopatológicos el padecimiento mental en niños/as con vulneración socioeconómica, refiriéndose a una niñez "otra", alejada de las expectativas sociales y definida por la peligrosidad (Barcala, 2019). Asimismo, respecto a las demandas de cambio de identidad de género, se observa que, en algunos casos, continúa operando una visión patológica para las niñeces trans más allá de los avances en materia de derechos humanos (Ministerio de Salud, 2020; Tajer, 2014).

El objetivo del presente artículo es introducir algunas consideraciones que hacen a la producción de subjetividad de las niñas, en particular, la dimensión histórico-social y las problemáticas actuales vinculadas a la violencia e identidad de género. Específicamente, nos proponemos problematizar algunos puntos que entendemos deben ser considerados al momento de la atención de las niñas en el ámbito de la salud. Partimos de considerar, a manera de hipótesis y con un sentido heurístico, que estos elementos producirían un impacto en el proceso de atención en tanto los imaginarios profesionales los contemplen o no.

En este sentido, proponemos una puntualización sobre distintas problemáticas que insisten y se despliegan como singularidades en el campo social respecto a las niñas y que nos permiten pensar una serie de vínculos con el proceso de saludenfermedad-atención. Si bien las niñas se incluyen entre los sectores sociales más 
desprotegidos que sufren una crónica desatención de sus necesidades (Lesbegueris, 2014), los estudios que indagan la atención en salud mental de estas problemáticas desde un enfoque de género, por el momento, son incipientes (Machado Zubeldía y Bardi, 2020; Bella y Borgiattino, 2016).

A partir de estas consideraciones, presentamos tres ejes problemáticos para los/as profesionales de la salud mental y para el sistema de salud:

- Existen vínculos entre los discursos y prácticas propias de la Modernidad en relación a las niñas y las estrategias de poder disciplinar y biopolíticas que los sostienen. Es necesaria una atenta vigilancia epistemológica sobre los saberes y prácticas disciplinarios y las desigualaciones que producen, por si posible impacto en el proceso de salud-enfermedad atención.

- La violencia constituye una problemática de la salud para las mujeres y una problemática sanitaria. Por ello, es necesario enunciar los modos en que se presentas las violencias de género contra las niñas y su posible vínculo con el proceso de salud-enfermedad-atención.

- La emergencia de las niñeces trans implica dar cuenta del cisexismo como sistema excluye y patologiza a las identidades trans, y del modo en que estas prácticas se presentan en el ámbito de la salud.

\section{Acerca de la dimensión histórico-social de las niñas}

La salud mental constituye un campo de saberes y técnicas amplio y complejo en el que coexisten corrientes teóricas diversas y antagónicas respecto a qué se entiende por niñez y qué aspectos se consideran relevantes para la indagación e intervención en salud (Stolkiner y Ardila Gómez, 2012). Entre ellas, son conocidas las disputas teóricas de las corrientes psicoanalíticas y las neurociencias respecto a los padecimientos mentales y las decisiones diagnósticas y terapéuticas en la niñez (Mantilla, 2014).

El psicoanálisis posee un lugar destacado en Argentina ya que constituye una corriente teórica hegemónica en la formación de los/as psicólogos/as (Dagfal, 2009). No obstante, en las últimas décadas se acude al crecimiento de la psiquiatría biológica (Cooper, 2005), que toma los desarrollos de las neurociencias cognitivas para el establecimiento de diagnósticos y abordajes en salud mental.

Por una parte, las neurociencias cognitivas centran su interés en el estudio de las características y funciones cerebrales con el fin de establecer bases biológicas de la cognición, los comportamientos y los trastornos mentales en niños/as (Escera, 2004). Aquí, las condiciones histórico-sociales no suelen contemplarse o son introducidas como un nivel de análisis que define a lo social como factor de influencia (Ochsner y Lieberman, 2001).

Por otra parte, el psicoanálisis centra su interés en el pensamiento, las defensas y conflictivas en la estructuración del psiquismo en la niñez (Janin, 2019). Respecto a 
la dimensión histórico-social, aporta grandes herramientas para pensar el psiquismo sin reproducir antinomias que homologan la psiquis a lo interior y la sociedad a un factor de influencia exterior (Fernández, 2007). No obstante, los estudios de psicoanálisis y género señalan que, en muchos casos, las conceptualizaciones psicoanalíticas tradicionales han opacado las articulaciones de género y poder en los devenires identitarios y sexuales y en los modos que adquieren los conflictos en la niñez (Lo Russo y Reid, 2020).

Por ello, en línea con estos desarrollos, consideramos necesario sostener una tensión entre los aspectos biológicos, la constitución del psiquismo y la dimensión históricosocial en la niñez. Esta última, pone en escena la capacidad creadora de los colectivos en la producción de sus realidades y formas de existir. Para nombrar esta capacidad creadora, Castoriadis (2010) establece la categoría de imaginario social y lo define como la creación histórico-social de significaciones imaginarias sociales, que otorgan sentidos comunes a los individuos y que se caracterizan por conducir las ideas y acciones de los colectivos en un determinado momento histórico-social (Fernández, 2007).

Por lo tanto, incorporar la dimensión histórico-social no se reduce a considerar la influencia de factores sociales en la niñez sino que se trata de dar cuenta de las formas histórico-sociales que adoptan la producción de esos/as niños/as y sus vínculos con las estrategias de poder-saber en determinada época.

Desde aquí, consideramos que la niña y la niñez no remiten a conceptos a-históricos y de validez universal sino, más bien, a una producción histórico-social (Ariès, 1987; Fernández, 2014; D'Agostino y Kreis, 2018). El advenimiento de la niña es producto de una serie de transformaciones en las creencias y en la vida cotidiana durante el surgimiento y consolidación de la sociedad capitalista en la Modernidad (Ariès, 1987; Donzelot, 2008). Los discursos, prácticas sociales y valores referidos a la niñez se produjeron en Europa y Estados Unidos y operaron como ideologías hegemónicas que alcanzaron a diversos países y a sectores sociales (Fernández, 2014).

La particularización de la niñez se vinculó con el despliegue de estrategias de poder que tomaron en consideración la vida. De acuerdo con los aportes de Foucault (2013), el poder sobre la vida se desarrolló a través de estrategias disciplinares y biopolíticas. Por una parte, el poder disciplinar se centró en el cuerpo como máquina, apuntó a su educación y al aumento de la utilidad y de la docilidad para la integración a los sistemas económicos. En este sentido, observamos que entre fines del siglo XVIII y principios del XIX, surgieron disciplinas que produjeron saberes y prácticas acerca de la niñez. La pedagogía, la psicología y la medicina se interesaron por las características sociales, emocionales, intelectuales y evolutivas de los/as niños/as, y estudiaron su especificidad en detalle. De esta manera, a través de una minuciosa descripción y análisis, comenzaron a delimitarse, en forma explícita e implícita, los parámetros aceptables, deseables y esperables del niño y de la niña.

Por otra parte, la biopolítica se centró en el cuerpo-especie como soporte de procesos biológicos. A partir de aquí, problemas como los nacimientos y la mortalidad, el 
nivel de salud, la duración de la vida y la longevidad pasaron al campo de control del saber y de intervención del poder (Foucault, 2013). Se focalizó en la salud, las maneras de alimentarse, de alojarse y las condiciones de vida. En cuanto a la niñez, las políticas buscaron favorecer el nacimiento y la supervivencia de la población infantil y disminuir las causas de mortalidad vinculadas a "accidentes domésticos" (Donzelot, 2008), producto de la ausencia de cuidados en las prácticas de crianza.

En este contexto, se fueron consolidando una serie de discursos y prácticas sobre la niñez, ancladas en significaciones imaginarias sociales que definieron al niño/a como un ser incompleto e incapaz que requiere de acompañamiento, de atención, de cuidados y de educación para asegurar su adaptación al medio social.

Sin embargo, no todos/as los/as niños/as responden históricamente a un universo común de significaciones ni se inscriben en las mismas prácticas. En el libro La invención de la niña, Fernández (1994) establece que el proceso de particularización de la niñez, como una etapa de la vida que requiere cuidados y educación, fue primero en niños que en niñas.

A diferencia de los niños, la particularización de las niñas no inició con el dispositivo escolar. Se desarrolló a partir de una educación moral que las instruía para el "desempeño óptimo" de la maternidad y de la vida conyugal. Para ello, las disciplinas promovieron una serie de cambios en las prácticas familiares anteriores, como la postergación de edad para el matrimonio y la prohibición de la maternidad en la niñez (Fernández, 2019).

La educación moral tenía dos objetivos: asegurar la virginidad hasta el matrimonio e instruir a las niñas para ser esposas dóciles y obedientes. Aquí, virginidad no aludía únicamente a la ausencia de relaciones sexuales antes del matrimonio, sino a todas aquellas "ignorancias" que garantizaban que el encuentro sexual dependa de la iniciativa y de la actividad del varón (Fernández, 2019). De esta manera, las niñas quedaron excluidas de experiencias de búsqueda activa de placer y de la exploración con el propio cuerpo. También, se las excluyó del saber sobre su cuerpo y de la nominación de sus genitales externos e internos. Se significó únicamente el útero, convertido en recinto de los/as hijos/as a engendrar (Giberti, 2009).

En el Segundo sexo, Beauvoir (1949/2009) describe cómo la niña se inserta desde pequeña en una trama de significaciones que confirman, a través de relatos, libros y juegos infantiles, la conyugalidad y la maternidad como destino. La niña será esposa, madre y abuela; cuidará su casa exactamente como lo hace su madre, y a sus hijos así como ella ha sido cuidada; tiene doce años y su historia ya está escrita en el cielo; la descubrirá día a día, sin hacerla jamás; es curiosa, pero se siente espantada cuando evoca esa vida cuyas etapas han sido ya todas previstas y hacia lo cual cada jornada la encamina ineluctablemente. (de Beauvoir, 1949/2009, p. 44)

Por lo tanto, observamos que la invención de la niña respondió a estrategias de saberpoder que consolidaron el lugar social de las mujeres como esposas y madres 
(Fernández, 2019). Ahora bien, los imaginarios sociales que situaban a la niña y a la mujer como seres frágiles, virginales, maternales y tutelados por figuras masculinas, era propia de los sectores burgueses de Europa y Estados Unidos. En los sectores populares, la particularización de las niñas demoró hasta comienzos del siglo XX. Hasta entonces, la intersección de clase social y género delimitó formas de vida ligadas a la explotación laboral, al trabajo doméstico, a la maternidad adolescente y a la prostitución (Fernández, 2014).

En América Latina, la particularización de la niñez posee coordenadas específicas. Los sentidos y los afectos modernos vinculados a los/as niños/as se consolidaron con la colonización europea del siglo XIX. En Argentina, la población infantil estaba lejos de responder a un universo de significaciones común. Los/as niños/as pertenecían a la población inmigrante del siglo XIX, a la población criolla y a sectores oligárquicos de la capital y de las provincias. Entre ellos/as existían grandes diferencias culturales y desigualdades sociales (Carli, 2018).

Aquí también, las prácticas de crianza y de socialización presentaron diferencias en función de los atravesamientos de clase social y género. En las niñas de los sectores medios, se priorizó la enseñanza de habilidades destinadas a un futuro casamiento "provechoso", como la lectura, la escritura y el aprendizaje de idiomas y de instrumentos musicales. Mientras tanto, las niñas de escasos recursos se insertaron en Sociedades de Beneficencia donde eran alfabetizadas y formadas en labores manuales como la costura, el bordado y el tejido. A su vez, las niñas de familias adineradas disponían de institutrices extranjeras que les enseñaban idiomas y lectura de textos religiosos (Lesbegueris, 2014).

El análisis genealógico, como herramienta que permite encontrar vínculos entre las narrativas teóricas y las condiciones histórico-sociales que las sostienen (Foucault, 2013), nos permite problematizar conceptualizaciones normativas que vinculan a las niñas con un ideal único. Asimismo, nos permite advertir que, en algunos casos, las diferencias entre niños y niñas constituyen desigualaciones de género en tanto se vinculan a estrategias de saber-poder que naturalizan y legitiman a las mujeres como un grupo social inferior desde la temprana edad (Fernández, 2019).

Al respecto, Meler (2009), referente de los Estudios de psicoanálisis y género argentinos en el campo de la psicopatología, señala que tradicionalmente los criterios de salud mental para las mujeres se homologaron a un modo de subjetivación femenino basado en la dependencia masculina, el desinterés, la auto postergación y la inhibición de la sexualidad y de la expresión hostil. A su vez, establece que, paradójicamente, la reproducción social de estos ideales femeninos constituyó un elemento determinante en la producción de malestares y estimuló la aparición de una serie de padecimientos como la histeria, considerada por la psiquiatría como una de las grandes epidemias femeninas del siglo XIX (Meler, 2009; Fernández, 2019). La equivalencia entre histeria y feminidad constituye un imaginario social sobre la sexualidad femenina que sostiene, con distintas tramas argumentales, su inferioridad (Fernández, 2019; Foucault, 2013). 
Lo anteriormente desarrollado nos permite sostener, en la posterior indagación, una vigilancia epistemológica respecto a la forma en que los/as profesionales de salud construyen discursos y prácticas sobre las niñas y su salud mental. Desde el enfoque de género en salud, consideramos que las desigualaciones de género, en intersección con otras diferencias fuente de inequidad, constituyen un problema de carácter político que es necesario contemplar a la hora de indagar las problemáticas y los abordajes en salud mental (Tajer, 2012).

\section{La violencia de género en niñas como problemática de salud}

En lo desarrollado hasta aquí, hemos visto como la particularización de las niñas se vinculó históricamente a estrategias de saber-poder y a universos de significaciones que naturalizaron las desigualaciones de género desde la temprana edad.

El enfoque de género en salud (Tajer, 2012) establece que las asimetrías sociales entre varones y mujeres determinan diferencialmente el proceso de salud-enfermedadatención. Estas asimetrías jerárquicas, articuladas con otras diferencias como edad, etnia y clase social, están presentes en la producción de problemáticas específicas en salud y en los modos de atención (Tajer, 2012). De este modo, se afirma que violencia de género es una problemática actual que puede tener un impacto en la salud de las niñas y que es necesario dilucidar a la hora de indagar las problemáticas y abordajes en el campo de la salud mental.

$\mathrm{Al}$ respecto, la OMS (2016) establece que la violencia de género es una problemática de derechos humanos y salud pública a nivel mundial, presente en diversos sectores socioeconómicos y culturales y en distintos momentos del ciclo vital de las feminidades. La Corte Interamericana de Derechos Humanos para prevenir, sancionar y erradicar la violencia contra la mujer (1994) define a la violencia de género como una violación de los derechos humanos basada en acciones o conductas que causan muerte, daño o sufrimiento físico, sexual o psicológico a mujeres en el marco de las asimetrías jerárquicas entre los géneros.

La violencia de género tiene graves consecuencias para la salud de las mujeres (Sen, et al. 2007). Asimismo, constituye una problemática sanitaria ya que implica una carga sustancial para los sistemas de salud, por ser un precedente relevante de consultas médicas y un factor que eleva el costo de la consulta externa (Tajer et al., 2014).

En la niñez, la violencia es una problemática presente en las consultas de salud que aparece como un desafío para los equipos en tanto requiere de un enfoque de trabajo interdisciplinario e intersectorial para su abordaje (Faraone, et. al., 2015). Investigaciones realizadas en el país indican que las demandas de salud mental muestran diferencias por género en la niñez. En este sentido, Bella y Borgiattino (2016) establecen que las internaciones en niñas en edad escolar, a diferencia de los niños varones, suelen estar motivadas por sospechas de abuso sexual infantil (Bella y Borgiattino, 2016). Asimismo, Zubeldía y Bardi (2020) señalan que las niñas constituyen un grupo de riesgo ya que suelen presentar una sintomatología menos 
disruptiva que la de los varones, lo que produce una detección tardía de sus malestares.

Es necesario entonces, dar cuenta de una caracterización que contemple estas violencias en el atravesamiento de género y edad, y visibilice distintas modalidades que sufren las niñas como sector social diferenciado al de los niños varones y las mujeres adultas, para intervenir cuando las mismas se presentan en el ámbito de la salud.

\section{Violencia doméstica}

Abarca desde formas de educación sostenidas en modelos autoritarios hasta el ejercicio de violencias, como el abuso físico y psicológico, el abandono o negligencia, la explotación y el abuso sexual. En Argentina, el Informe estadístico sobre situaciones de violencia doméstica (Oficina de Violencia Doméstica de la Corte Suprema de la Nación, 2019), establece que en 2018, el 32\% de las personas afectadas evaluadas fueron niños, niñas y adolescentes (NNyA). En las niñas y adolescentes, las formas de violencia más recurrentes son la violencia sexual, psicológica, simbólica y física. Como dato relevante, se establece que en la mayoría de los casos, las personas denunciadas son varones con un vínculo filial con las niñas. Recién entre los 14 y los 17 años aumenta el porcentaje de denuncias hacia personas que no pertenecen al ámbito familiar de niñas y adolescentes, como es el caso de los vínculos de noviazgo.

\section{Abuso sexual infantojuvenil}

Toporosi (2018) lo define como una práctica que viola la ley y los tabúes sociales. Consiste en la convocatoria de un adulto hacia un/a niño/a a participar de actividades sexuales que no puede comprender y, que, por ende, tampoco puede consentir desde una posición de sujeto (Toporosi, 2018). A nivel mundial, según un informe de la Organización Mundial de la Salud (2012), una de cada cinco mujeres y una de cada trece varones han declarado haber sufrido abusos sexuales durante su infancia. En el mismo sentido, el Estudio global elaborado por el Fondo de las Naciones Unidas para la Infancia (UNICEF) en el año 2014 estima que más de una de cada diez niñas sufrieron abuso sexual en su infancia. En Argentina, los datos obtenidos por el Programa Las Víctimas contra las Violencias demuestran que el $77,9 \%$ de los/as NNyA víctimas de violencia sexual son de género femenino. Esta diferencia de género se mantiene para todos los grupos etarios y se profundiza en el caso de las adolescentes. En el 76,8\% de los casos, los agresores pertenecen al entorno cercano de la víctima (familiar o no).

\section{Matrimonio infantil o casamiento precoz y maternidad forzada}

El matrimonio infantil se define como cualquier unión legal, o habitual, entre dos personas, de las cuales una o ambas están por debajo de 18 años de edad. Afecta principalmente a niñas y adolescentes, exponiéndolas ante situaciones de violencias, embarazos forzados y abortos inseguros (Mendoza Tascón et. al, 2016). 
Más de sesenta millones de mujeres entre 20 y 24 años en todo el mundo se casan antes de llegar a los 18 años (Mendoza Tascón et. al, 2016). En Argentina, el informe del Equipo Latinoamericano de Justicia y Género (ELA) y de la Fundación para el Estudio e Investigación de la Mujer (FEIM) evidencia que existe una mayor vulnerabilidad de las niñas al casamiento precoz (2017). En 2010, el Censo Nacional de Población y Vivienda identificó que 341 mil niños/as y adolescentes entre 14 y 18 años conviven con mayores de edad, por unión consensual o legal. Del total, $231 \mathrm{mil}$ son mujeres que, a diferencia de los varones, conviven con hombres entre 10 y 20 años mayores (Instituto Nacional de Estadística y Censos, 2010).

El embarazo y la maternidad forzada afectan la salud integral de las niñas y la inserción en el ámbito social, escolar y recreativo (UNICEF, 2018). Según el informe Embarazo y maternidad en adolescentes y menores de 15 años (UNICEF, 2018), en Argentina cada 3 horas una niña de entre 10 y 14 años es forzada a gestar, parir y criar. Asimismo, el $80 \%$ de los casos de embarazos son producto de abuso sexual intrafamiliar (UNICEF, 2018).

\section{Explotación sexual y trata de personas}

Giberti (2014) define a la trata de personas como una forma de esclavitud moderna y un fenómeno mundial provocado por la demanda, la violencia de género, el desempleo, la pobreza, la discriminación y la escasa acción de los poderes públicos. Abarca abusos como la explotación sexual, el trabajo forzoso o la explotación en la mendicidad. La explotación sexual comercial infantil es un ejercicio de poder que mercantiliza el abuso sexual de $\mathrm{NNyA}$ con el fin de obtener ganancia económica o provecho sexual. La explotación incluye relaciones sexuales remuneradas, pornografía, tráfico y venta de niños/as, entre otras formas. Los explotadores son, en la mayoría de los casos, hombres adultos y las víctimas son niñas y, en menor medida, niños (Gutiérrez et al., 2008).

Según el informe de la Oficina de Naciones Unidas contra la Droga y el Delito (2018), en América Central y el Caribe, el 55\% de las víctimas son niñas y el 25\%, mujeres adultas. En América del Sur, de las 3 mil víctimas identificadas, 31\% son niñas y $51 \%$, mujeres. En Argentina no existen estadísticas oficiales sobre el número de víctimas de trata sexual de personas. No obstante, acorde a datos otorgados por ELA, entre 2007 y 2009, se comprobó la existencia de más de 600 niñas y adolescentes víctimas de trata, pertenecientes, en general, a sectores de vulneración socioeconómica (Lesbegueris, 2014).

\section{Violencia y exclusión escolar}

En el área de la educación, Fernández (2014) sostiene que nos encontramos en un espejismo de igualdad ya que los/as niños/as y adolescentes de sectores medios urbanos han logrado acceder a la escolarización en masa; no obstante, las prácticas educativas naturalizan y reproducen roles y prácticas discriminatorias para las mujeres. En las escuelas, la producción y la reproducción de los poderes de género continúan siendo desigual, ya que la palabra y la representatividad es 
predominantemente masculina (Fernández, 2014).

Consideramos que las distintas manifestaciones de la violencia de género en niñas constituyen un problema social y de salud que incumbe a las instituciones y dispositivos de atención en salud mental infantojuveniles. Ampliar la visión sobre estas problemáticas y sus efectos en la salud mental de las niñas que son asistidas requiere de la presencia de discursos y prácticas en salud mental que contemplen que el proceso de salud-enfermedad-atención implica el entramado de atravesamientos socio-económicos, políticos y culturales y de género.

\section{Deconstruir y descisexualizar sentidos a la hora de pensar las problemáticas de las niñas}

Tajer (2017) establece que actualmente acudimos a cambios en los imaginarios sociales acerca de la sexualidad y el género que producen nuevas prácticas subjetivas y vinculares, junto con legislaciones que las legitiman socialmente, como la Ley Nacional de Identidad de Género n²6743 (2012). En el campo de la niñez, estos cambios posibilitan la constitución de identidades, en las que identidad de género y sexo adjudicado al nacer, no necesariamente coinciden.

La noción de identidad de género proviene de los hallazgos de John Money en el campo de la psiquiatría infantil a partir del trabajo con niños/as intersex (Money y Erhard, 1982). Estas niñeces representaban ciertos desafíos para la medicina ya que sus órganos genitales no podían clasificarse fácilmente como femeninos o masculinos (Mattio, 2012). Por ello, el concepto de identidad de género se estableció para referirse a la convicción subjetiva de los/as niños en la adopción de la identidad femenina o masculina desde la temprana infancia con independencia de la maduración biológica ulterior (Dio Bleichmar, 1996).

Posteriormente, el concepto de género fue adoptado por el feminismo académico y militante de los años 70 como una herramienta fundamental para cuestionar la organización social de las relaciones entre los sexos (Mattio, 2012). A partir aquí, el género se refiere al conjunto de prácticas, símbolos y valores sociales que establecen la construcción desigual de esferas masculinas y femeninas basadas en la diferencia sexual anátomo-fisiológica.

Butler (2007) cuestiona esta noción de género proveniente de la teoría feminista de fines del siglo XX. Considera que definir al género como la construcción cultural variable del sexo implica sostener que este último es un atributo biológico inmutable y predeterminado. Para la autora, la construcción del sexo, como la del género, es cultural ya que su categorización binaria y discreta es producto de un régimen de sexualidad histórico y específico (Butler, 2007; Foucault, 2013).

Desde allí, define al género como el aparato de construcción cultural a través del cual se produce tanto la naturalización de lo femenino y lo masculino como su deconstrucción. En palabras de Butler, "cuando nos referimos al 'género en disputa 
o problematización del género’ (...) ya sea el transgénero o el cruce de géneros, estamos sugiriendo que el género tiene una forma de desplazarse más allá de lo binario naturalizado" (2018, p. 70).

Estos desarrollos nos permiten una mayor amplitud a la hora de pensar a las niñas. A partir de aquí, consideraremos algunas problemáticas de las niñas trans distintas a las de las niñas cis. El término cis es creado por la comunidad trans en los años 90 y utilizado por activistas e investigadores/as para referirse a aquellas personas cuya identidad de género asignada al momento de nacer se corresponde con la identidad de género autopercibida (Radi, 2020). Esta nominación supone un cambio de paradigma ya que quienes nombran son aquellos/as que históricamente han sido nombrados y categorizados. Asimismo, complejiza la categoría de género, la cual ya no se reduce únicamente a mujeres y varones cis.

Lo anterior implica reconocer que la identidad de género en la niñez se encuentra en proceso de constitución. Como sostienen los estudios de psicoanálisis y género, la identidad de género se constituye a partir la interacción de las propuestas identificatorias (conscientes e inconscientes) emanadas en los cuidados primarios y la apropiación activa por parte del niño/a (Tajer, 2017). Es decir, la identidad de género en las niñas deviene de una relación particularizada con otros/as que encarnan las instituciones de la sociedad. Hablar de identidades en la infancia (trans o cis) no niega la posibilidad de futuros cambios; no obstante, para las niñeces trans, permite nombrar y legitimar la emergencia histórico-social de otras niñeces posibles.

La niñez transgénero es una forma social que emerge en la década del 90 y se visibiliza a comienzos del siglo XXI, a partir del activismo de madres, padres y educadores/as (Halberstam, 2019). Hasta entonces, las demandas de cambio de identidad se consideraban una problemática exclusivamente adulta. Para las niñeces que expresaban anhelos de ser reconocidas como personas de otro género, el único abordaje posible era el diagnóstico y tratamiento por presuntas perturbaciones psíquicas. Actualmente, la patologización de la niñez continúa y se profundiza; no obstante, en algunos contextos, comienza a emerger la niñez trans como posible de ser escuchada y visibilizada.

En el campo de la salud mental, si bien la Ley Nacional de Salud Mental n²6.657 (2010) establece que en ningún caso puede hacerse diagnóstico sobre la base exclusiva de identidad y elección sexual, la emergencia de estas niñeces ha suscitado una serie de debates. Las inquietudes se vinculan con la "aparición temprana" de la demanda de cambio de identidad en la niñez o a la diferenciación entre problemáticas relativas a la identidad $\mathrm{y}$ otras vinculadas con trastornos psicopatológicos (Tajer et al., 2016).

Por ello, consideramos importante la construcción de propuestas que apunten a visibilizar el cisexismo en el sistema de salud (Millet, 2018). Para Millet (2018), el cisexismo es un sistema hegemónico basado en la creencia de que la vida, los cuerpos, las identidades y los deseos de las personas trans, valen menos que los de las personas cis. En contextos cisexistas, las vidas de aquellas personas que se identifican 
con el género asignado al nacer tienden ser vistas como normales y saludables, en oposición a la existencia de las personas trans, entendidas como anormales y patológicas (Radi, 2020).

De este modo, ser o parecer trans posee consecuencias materiales y simbólicas que determinan formas de exclusión y barreras en el acceso a la salud (Millet, 2018). En muchas oportunidades, las personas trans no consultan ni permanecen en los dispositivos de salud debido a la ausencia de profesionales que alojen sus necesidades sin ejercer violencias (Millet, 2018). En efecto, el Informe sobre niñeces trans (ACIL, 2019) establece que la mayoría de las familias estudiadas vincularon el sentir de sus hijos/as a un malestar o padecer y que un $35 \%$ buscó ayuda en un profesional de la salud, sin lograr experiencias satisfactorias.

A su vez, como mencionamos anteriormente, la medicalización y patologización de las identidades continúa siendo una práctica médica habitual. La medicalización implica la definición de las problemáticas de los/as niños/as, como enfermedades, trastornos o desórdenes, y la utilización exclusiva de la intervención médica para el tratamiento (Stolkiner y Ardila Gómez, 2012). En las demandas de cambio de identidad de género, se observa que es usual la utilización de diagnósticos como "trastorno de identidad de género" y "disforia de género" en la niñez, y el establecimiento abordajes constituidos por prácticas correctivas de reforzamiento de los roles tradicionales de género (Ministerio de Salud, 2020).

Estos abordajes invisibilizan una concepción integral de la salud mental como proceso determinado por componentes culturales, históricos, sociales, económicos, psicológicos y biológicos que necesariamente requieren de una dinámica de construcción social vinculada con la concreción de los derechos humanos (LNSM, 2010).

A partir de estas consideraciones, observamos una serie de tensiones entre procesos socio-históricos instituyentes e instituidos referidos a las niñas. Como desarrollamos al comienzo, la particularización de las niñas responde a un proceso histórico social de desigualación que parte de las diferencias biológicas para justificar desigualdades sociales. No obstante, al mismo tiempo, los cambios histórico-sociales actuales generan condiciones de posibilidad para que en determinados contextos emerjan producciones subjetivas, con posicionamientos objetivos-subjetivos de autonomía de género, reflejados en la elección de identidades y de proyectos vitales.

Por ello, desde el campo de la salud mental resulta importante alojar estas nuevos existenciarios, a partir de considerar que la producción de subjetividad no se limita a la constitución del sujeto psíquico sino que alude también a un proceso en permanente transformación que se construye en el entre, con otros/as que encarnan las instituciones de la sociedad. En este sentido, no existe una sola forma de devenir niña ya que las coordenadas de producción de subjetividad articulan dimensiones deseantes, políticas, históricas, simbólicas, psíquicas, sexuales, entre otras (Fernández, 2007). 
Desde aquí, el desafío consiste en la invención de respuestas en salud basadas en la deconstrucción y decisexualización de saberes, de prácticas y de estrategias de abordajes que incorporen, desde un enfoque de género en salud, la diversidad en cuanto a la identidad y a las expresiones de género en la niñez.

\section{A modo de conclusión}

Nos hemos propuesto la puntualización de distintas problemáticas que insisten y se despliegan como singularidades en el campo social, como un modo de visibilizar y poner en tensión prácticas de desigualación que se presentan en el ámbito de la salud y la salud mental. Observamos que las desigualaciones de género persisten en la actualidad y producen una serie de violencias que podrían repercutir en el proceso de salud-enfermedad-atención.

Consideramos que las violencias constituyen una problemática sanitaria que es necesario continuar indagando en los dispositivos de salud mental infantojuveniles. Entre ellas, señalamos la particularización de las niñas, vinculada al sostenimiento de universos de sentido y estrategias de poder que naturalizan formas de inferiorización de las feminidades.

No pretendemos ser taxativas en esta descripción, solo aportar algunos puntos de reflexión como un elemento para la interrogación de las propias prácticas. Las problemáticas enunciadas constituyen un desafío para los/as profesionales y para el sistema de salud. Por ello, sostenemos la importancia de indagar los sentidos y prácticas acerca del proceso de salud-enfermedad-atención para estas niñeces, como elemento para la condición de posibilidad en la invención de nuevas significaciones, como formas de resistencias a estas discriminaciones.

\section{Referencias}

Ariès, P. (1960/1987). El niño y la vida familiar en el Antiguo Régimen. Tauros.

Asociación Civil Infancias Libres (2019). Informe sobre niñeces trans. HTTPS://ES.SCRIBD.COM/DOCUMENT/ 426261374/INFORME-INFANCIAS-LIBRES-JULIO-2019

Barcala, A. (2019). Discursos en salud mental: construcción de subjetividades en la niñez en la ciudad de Buenos Aires. Interface-Comunicação, Saúde, Educação, 23, 1-17. HTTPs://DOI.ORG/ 10.1590/INTERFACE. 180464

Bella, M. E, y Borgiattino, V. (2016). Demand for hospitalization due to psychosocial causes in a pediatric hospital. Archivos Argentinos de Pediatría, 114(3), 252-257. HTTPS://DX.DOI.ORG/ 10.5546/AAP.2016.252
Butler, J. (2007). Sujetos de sexo/género/deseo. En El género en disputa. El feminismo y la subversión de la identidad (pp. 46-100). Paidós.

Butler, J. (2018). El reglamento del género. En Deshacer el género (pp. 67-88). Paidós.

Carli. S. (2018). La invención de la infancia moderna. Domingo Faustino Sarmiento y la escuela pública. En Niñez, pedagogía y política: transformaciones de los discursos acerca de la infancia en la historia de la educación argentina entre 1880 y 1955 (pp. 35-58). Miño y Dávila Editores.

Castoriadis, C. (2010). El imaginario social y la institución. Lo histórico social. En La institución imaginaria de la sociedad (pp. 269-351). Ensayo. 
Cooper, R. (2005). Classifying madness. A philosophical examination of the Diagnostic and Statistical Manual of Mental Disorders. Springer.

Corte Interamericana de Derechos Humanos (1994) Convención Interamericana para Prevenir, Sancionar y Erradicar la Violencia contra la Mujer. нтTPs:// WWW.OAS.ORG/ES/MESECVI/CONVENCION.ASP

Dagfal, A. (2009). Entre París y Buenos Aires: La invención del psicólogo (1942-1966). Paidós.

D'Agostino, A. M. E. y Kreis, M. M. (2018) Consideraciones acerca de la accesibilidad y el derecho a la salud en la niñez y la adolescencia. Anuario Temas en Psicología, 4, 163-177. нттP:// SEDICI.UNLP.EDU.AR/HANDLE/10915/72989

de Beauvoir, S. (1949/2009). Infancia. En El segundo sexo (pp. 87-116). Cátedra.

Dio Bleichmar, E. (1996). Feminidad/masculinidad. Resistencias en el psicoanálisis al concepto de género En M. Burín y E. Dio Bleichmar (Eds.), Género, psicoanálisis, subjetividad (pp. 100.-139). Paidós.

Donzelot, J. (2008). La conservación de los hijos. En La policía de las familias (pp. 13-48). Nueva Visión.

Equipo Latinoamericano de Justicia y Género (ELA) y Fundación para Estudio e Investigación de la Mujer (FEIM) (2017). Informe al Comité de los Derechos del Niño. HTTP://WWW.ELA.ORG.AR/A2/ INDEX.CFM?MUESTRA $\&$ APLICACION $=\mathrm{APP} 187 \& \mathrm{CNL}=4$ $\&$ OPC $=50 \&$ CODCONTENIDO $=2948 \&$ PLCONTAMPL $=12$

Escera, C. (2004). Aproximación histórica y conceptual a la Neurociencia Cognitiva. Cognitiva, 16(2), 141-61.

Faraone, S. A., Valero, A. S., Bianchi, E. (2015). "Salir del pantano": Violencias, infancia y equipos de salud: claves para pensar dispositivos y acciones en salud mental. Derecho y Ciencias Sociales, 12, (4)-2015, 70-88. HTTPS://DIALNET.UNIRIOJA.ES/ SERVLET/ARTICULO?CODIGO $=5164612$

Fernández, A. M. (1994). La invención de la niña. UNICEF.

Fernández, A. M. (2007). Haciendo Met-hodos, En Las lógicas colectivas. Imaginarios, cuerpos $y$ multiplicidades. Biblos.

Fernández, A. M. (2014). Historia de las infancias. En Las lógicas sexuales: amor, política y violencias (pp. 73-100). Nueva Visión.

Fernández, A. M. (2019). ¿Historia de la histeria o histeria de la historia? En La mujer de la ilusión (pp. 59-93). Paidós
Fondo de las Naciones Unidas para la Infancia (2014). Abuso sexual contra niños, niñas y adolescentes Una guía para tomar acciones y proteger sus derechos. HT TPS://WWW. UNICEF.ORG/ARGENTINA/SITES/ UNICEF.ORG.ARGENTINA/FILES/2018-04/PROTECCIONABUSOSEXUAL_CONTRA_NNYA-2016.PDF

Fondo de Naciones Unidas para la Infancia (2018). Abusos sexuales y embarazo forzado en la niñez $y$ adolescencia. HTTPS://WWW.UNICEF.ORG/ ARGENTINA/INFORMES/ABUSOS-SEXUALES-YEMBARAZO-FORZADO-EN-LA-NI\%C $3 \%$ B 1EZ-YADOLESCENCIA

Fondo de Naciones Unidas para la Infancia Argentina (2019). Un análisis de los datos del programa "Las Víctimas Contra las Violencias" 2018-2019. HTTPS:/ /WWW.UNICEF.ORG/ARGENTINA/MEDIA/6776/FILE/ DATOS\%20\%22LAS\%2OV\%C3\%ADCTIMAS\%2OCONT RA\%2OLAS\%2OVIOLENCIAS\%22\%202018-2019.PDF

Foucault, M. (2013). El dispositivo de la sexualidad. En Historia de la sexualidad. 1. La voluntad del saber (pp. 75-126). Siglo XXI.

Giberti, E. (2009). Niña. En S. B. Gamba (ed.), Diccionario de Estudios de Género y Feminismos (pp. 234-236). Biblos.

Giberti, E. (2014). La trata de personas: el desvalimiento en el crimen organizado. Desvalimiento Psicosocial, 1(1). HTTP:// DSPACE.UCES.EDU.AR: $8180 /$ XMLUI/HANDLE/ $123456789 / 2841$

Gutiérrez, R., Vega, L., y Rodríguez, E. M. (2008). Problemas y dilemas éticos en la investigación de la explotación sexual comercial de niñas y niños. Salud Mental, 31(5), 403-408. нттр:// W W W. S C I E L O . O $\quad$ R G . M X / SCIELO.PHP?SCRIPT $=$ SCI_ART TEXT\&PID $=$ SO 185 33252008000500008

Halberstam, J. (2019). Trans: un informe rápido y extravagante de la variabilidad de género en el siglo XXI y más allá. En M. L. Seone (Ed.), Los mil pequeños sexos. Intervenciones críticas sobre políticas de género y sexualidades (pp. 120-132). EDUNTREF.

Instituto Nacional de Estadística y Censos (2010). Censo de población y vivienda. HTTPS://WWW.INDEC.GOB.AR/ INDEC/WEB/NIVEL4-TEMA-2-41-135

Janin, B. (2019). El abuso sexual y los diagnósticos invalidantes. En Infancias $y$ adolescencias patologizadas. La clínica psicoanalítica frente al arrasamiento de la subjetividad. Noveduc.

Lesbegueris, M. (2014). Niñas en situación de vulnerabilidad: con lo que no se juega. En iNiñas jugando! Ni tan quietas ni tan activas (pp. 103-124). Editorial Biblos. 
Ley Nacional de Identidad de Género n²6743, República Argentina (2012). нттP:// SERVICIOS.INFOLEG.GOB.AR/INFOLEGINTERNET/ ANEXOS/195000-199999/197860/NORMA.HTM

Ley Nacional de Salud Mental n²6.657, República Argentina (2010). HTTP:// SERVICIOS.INFOLEG.GOB.AR/INFOLEGINTERNET/ ANEXOS/175000-179999/175977/NORMA.HTM

Lo Russo, A y Reid, G. (2020). Sobre género y diversidades sexuales en las adolescencias. En S Ferreiro dos Santos (Ed.), Clínica con adolescentes. Problemáticas contemporáneas. Editorial Entreideas.

Machado Zubeldía, M. y Bardi, D. C. (2020). Los estereotipos de género en la construcción subjetiva de las niñas: su relación con las derivaciones a servicios de salud mental. Anuario de Investigaciones, 27, 283-291. НTTP:// W $\quad \mathrm{W} \quad \mathrm{W}, \mathrm{P} \quad \mathrm{S}$ I, $\mathrm{U}$ B $\mathrm{A}, \mathrm{A}$ R INVESTIGACIONES.PHP?VAR=INVESTIGACIONES/ REVISTAS/ANUARIO/TRABAJO.PHP\&ID $=1145$

Mantilla, J. (2014). Psicoanálisis y neurociencias. Contornos de un debate vigente en la cultura psi argentina. Astrolabio, 12, 173-192. HTTPs:// REVISTAS.UNC.EDU.AR/INDEX.PHP/ASTROLABIO/ ARTICLE/VIEW/5357

Mattio, E. (2012). ¿De qué hablamos cuando hablamo de género? Una introducción conceptual. En J. M. Morán; S. Faúndes; M. C. Ruata y J. M. Vaggione (Eds.) Desigualdades y derechos. Reflexiones en torno a los derechos sexuales y reproductivos ( $\mathrm{pp}$. 85-103). Ciencia, Derecho y Sociedad.

Meler, I. (2009). Género y salud mental. Revista Topía. H T TPS:// WWW.TOPIA.COM.AR/ARTICUL OS/ G\%C3\%A9NERO-Y-SALUD-MENTAL

Mendoza Tascón, L. A., Claros Benítez, D. I., Mendoza Tascón, L. I., Peñaranda Ospina, C. B., Arias Guatibonza, M. D., Carrillo Bejarano, J. H. y Sarria Henao, Z. (2016). Matrimonio infantil: un problema social, económico y de salud pública. Revista Chilena de Obstetricia y Ginecología, 81(3), 254-261. HTTPS://DX.DOI.ORG/10.4067/S071775262016000300013

Millet, A. (2018). Barreras en la accesibilidad de personas trans de la ciudad autónoma de Buenos Aires a los tratamientos por uso problemático de sustancia. Programa de Becas de Investigación Hospital Nacional en Red, Especializado en Salud Mental y Adicciones Lic. Laura Bonaparte. HTTPS:/ /WWW.ARGENTINA.GOB.AR/SITES/DEFAULT/FILES/ 5._INFORME_FINAL_-_AN_MILLET.PDF

Ministerio de Salud (2020). Atención de la salud integral de personas trans, travestis y no binarias. HTTPS://BANCOS.SALUD.GOB.AR/SITES/DEFAULT/ FILES/2020-10/GUIA-SALUD-PERSONAS-TRANSTRAVESTIS-NOBINARIAS.PDF
Money, J. y Erhard, A. (1982). Desarrollo de la sexualidad humana (diferenciación y dimorfismo de la identidad de género). Morata.

Oficina de Naciones Unidas contra la Droga y el Delito (2018). South America. HTtP://Www.UNODC.ORG/ DOCUMENTS/DATA-AND-ANALYSIS/GLOTIP/2018/ GLOTIP_2018_SOUTH_AMERICA.PDF

Oficina de Violencia Doméstica de la Corte Suprema de la Nación (2019). Informe estadístico sobre niñas, niños y adolescentes afectados por situaciones de violencia doméstica. HTTPS://WwW.CIJ.GOV.AR/ NOTA-36159-LA-CORTE-SUPREMA-PUBLIC--UNINFORME-SOBRE-NI-AS--NI-OS-Y-ADOLESCENTESAFECTADOS-POR-VIOLENCIA-DOM-STICA.HTML

Organización Mundial de la Salud (2012). Ocultos a plena luz. Un análisis estadístico de la violencia contra los niños. HTTPS://WWW.UNICEF.ORG/ OCULTOS-A-PLENA-LUZ.PDF

Organización Mundial de la Salud (2016). Atención de salud para las mujeres que han sufrido violencia de pareja o violencia sexual. HTTPS://WWW.WHO.INT/ REPRODUCTIVEHEALTH/PUBLICATIONS/VIOLENCE/ VAW-CLINICAL-HANDBOOK/ES/

Ochsner, K. N. y Lieberman, M. D. (2001). The emergence of social cognitive neuroscience. American Psychologist, 56, 717-734.

Radi, B. (2020). Epistemología del asterisco: una introducción sinuosa a la epistemología trans. En D. Maffía, A. Moreno Sordá, Y. Espinosa Miñoso y B. Radi (Eds.), Apuntes epistemológicos (pp.107-121). UNR.

Sen, G., Östlin, P. y Asha G. (2007). La inequidad de género en la salud: desigual, injusta, ineficaz e ineficiente. Organización Panamericana de la Salud. HTTPS://WWW.PAHO.ORG/HQ/DMDOCUMENTS/2007/ LA\%2OINEQUIDAD_DE_GENERO_EN_LASALUD_DESI GUAL_INIUSTA_INEFICAZ_E_INEFICIENTE.PDF

Stolkiner, A. y Ardila Gómez S. (2012). Conceptualizando la Salud Mental en las prácticas: consideraciones desde el pensamiento de la medicina social/salud colectiva latinoamericanas. Revista Argentina de Psiquiatría, 23, 57-67.

Stolkiner, A. (2019). ¿Qué es escuchar a un niño? Escucha y hospitalidad en el cuidado en salud. En Miguel Angel Tollo (Ed.), Escuchar las infancias. Alojar singularidades y restituir derechos en tiempos de arrasamientos subjetivos. Noveduc.

Tajer, D. (Ed.). (2012). Género y salud: las políticas en acción. Lugar.

Tajer, D., Reid, G. y Gaba, M. (2014). Impacto de la violencia de género en la salud de las mujeres: una investigación en la Ciudad de Buenos Aires. Género y Salud en Cifras, 12(2), 11-26. 
Tajer, D., Reid, G., Salvo Agoglia, L. y Lo Russo, A. (2016). Identidad de género y salud sexual reproductiva en las consultas de adolescentes en servicios de salud de la ciudad de Buenos Aires. Anuario de Investigaciones, 23, 217-226. нттр:// WWW.PSI.UBA.AR/INVESTIGACIONES/REVISTAS/ ANUARIO/TRABAJOS_COMPLETOS/23/TAJER.PDF

Tajer, D. (2017). Algunas consideraciones éticas y clínicas sobre las infancias trans. En I. Meler (Ed.), Psicoanálisis y género. Escritos sobre el amor, el trabajo, la sexualidad y la violencia (pp. 277-294). Paidós.

Toporosi, S. (2018). En carne viva. Abuso sexual infantojuvenil. Topía Editorial. 